\title{
RISCO DE MORRER NO PRIMEIRO ANO DE VIDA ENTRE FAVELADOS E NÃO FAVELADOS NO MUNICIPIO DE PORTO ALEGRE, RS (BRASIL), EM 1980
}

Airton Fischmann*

José Joaquim de Lima Guimarães*

\begin{abstract}
FISCHMANN, A. \& GUIMARÃES, J.J. de L. Risco de morrer no primeiro ano de vida entre favelados e não favelados no município de Porto Alegre, RS (Brasil), em 1980. Rev.Saúde públ., S.Paulo, 20: 219-26, 1986.
\end{abstract}

RESUMO: Foram apresentados o risco relativo (RR) e o risco atribuivel percentual (RAP) ao fator favelado de morrer, no primeiro ano de vida, em quatro setores de Porto Alegre, RS (Brasil), em 1980. O risco relativo médio de morrer no primeiro ano de vida foi de 2,4 a 3,62 vezes maior para o favelado, considerado um intervalo de confiança de $95 \%$. O RAP ao favelado de morrer no primeiro ano de vida variou de $23,2 \%$ a $33,0 \%$ considerado um intervalo de confiança de $95 \%$. O estudo dos cinco principais grupos de causas revelou que com exceção dos óbitos por anomalias congênitas, o risco reiativo por essas causas foi sempre superior para o favelado; 1,8 vezes maior para afecçōes perinatais, 5,9 para a doença infecciosa intestinal, 6,1 para pneumonia e gripe e 8,0 para septicemia. Houve setores, como o intermediário sul, em que o risco relativo de morrer por septicemia foi 18,2 vezes maior para o favelado. Quanto ao risco atribuível, verificou-se que em média $28 \%$ da mortalidade infantil é atribuível a $18 \%$ de favelados. Considerados os principais grupos de causas, o RAP ao fator favelado foi de $12,8 \%$ para a mortalidade perinatal, $47,7 \%$ para doença infecciosa intestinal, $48,7 \%$ para pneumonia e gripe e $56,7 \%$ para septicemia. Mesmo levando em conta que o problema social e económico é o principal determinante do fato de um indivíduo ser favelado, recomenda-se verificar a qualidade da assistência à saúde prestada às populaçōes faveladas, pois é possível obter-se reduçāo de morbi-mortalidade por aquelas causas, através da aplicação de cuidados médicos adequados, dirigidos prioritariamente às populaçбes em piores condjçōes de vida.

UNITERMOS: Risco. Mortalidade infantil. Morbidade. Fatores sócio-econômicos.

\section{INTRODUÇÃO}

0 enfoque de risco é uma filosofia de trabalho de caráter essencialmente preventivo e é fundamento epidemiológico para a programação e regionalização da assistência à saúde. Constitui uma oportunidade para integrar a clinica com a epidemiologia, a administração e o planejamento em saúde. Também fa. vorece um melhor aproveitamento dos recursos ao permitir a sua adequação às necessidades, evitando acréscimo paralelo dos gastos de saúde.

Este enfoque vem sendo adotado em vários países e regiões, e inúmeros são os trabalhos publicados sobre o assunto, a nivel mundial. No entanto, muito pouco tem sido publicado no Brasil a este respeito, podendo-se citar o trabalho de Sarue e col.4, traduzido e adaptado, que aborda diferentes fatores de risco de mortalidade perinatal, a partir dos dados oriundos da Maternidade de Vila Nova Cachoeirinha, em São Paulo**.
A necessidade de fornecer subsidios para a pro. gramação de saúde, a partir de estudos de populações sujeitas ao maior risco de morrer, levou os autores a calcular o risco relativo (RR) do favelado de morrer no primeiro ano de vida e o risco atribuí. vel percentual (RAP) ao fator favelado na mortali. dade infantil de Porto Alegre, RS em 1980, a partir de dados já existentes.

\section{METODOLOGIA}

Vários métodos estatísticos têm sido desenvolvidos para medir o grau de associação em estudos epidemiológicos 3 . O risco relativo mede a associação entre a característica ou fator e a doença e pode ser descrito, como:

$$
R R=\frac{\text { Coeficiente de incidência da doença entre expostos }}{\text { Coeficiente de incidência da doença entre não expostos }}
$$

\footnotetext{
* Da Secretaria de Saúde e do Meio Ambiente do Rio Grande do Sul - SSMA - Av. Borges de Medeiros, 1501 - 5 9 andar, sala 2 - 90000 - Porto Alegre - RS - Brasil.

* Diagnóstico de Situação Perinatal da Maternidade Escola de Vila Nova Cachoeirinha, elaborado por Antonio Suzart Andrade - Dados inéditos.
} 
FISCHMANN, A. \& GUIMARĀES, J.J. de L. Risco de morrer no primeiro ano de vida entre favelados e não favelados no município de Porto Alegre, RS (Brasil), em 1980. Rev. Saúde públ., S. Paulo, $20: 219-26,1986$.

Considerando o quadro seguinte

\begin{tabular}{lccc}
\hline $\begin{array}{l}\text { Característica } \\
\text { ou Fator }\end{array}$ & $\begin{array}{c}\text { Doente } \\
(\text { Caso })\end{array}$ & $\begin{array}{c}\text { N Doente } \\
\text { Controle }\end{array}$ & Total \\
\hline c/fator & $a$ & $b$ & $a+b\left(m_{1}\right)$ \\
s/fator & $c$ & $d$ & $c+d\left(m_{2}\right)$ \\
\hline \multicolumn{1}{c}{ Total } & $a+c\left(n_{1}\right)$ & $b+d\left(n_{2}\right)$ & $a+b+c+d N$ \\
\hline
\end{tabular}

O risco relativo pode ser medido de duas formas:
Coeficiente de incidência entre
1. $\mathrm{RR}=$ expostos
Coeficiente de incidência entre
não expostos
$=\frac{\frac{a}{a+b}}{\frac{c}{c+d}}$
ou
2. pode ser estimado em estudos retrospectivos pela razão de produtos cruzados
$\frac{a x d}{b x c}$

Neste caso, assume-se que:

a) a freqüência do evento na população é muito pequena, ou

b) trata-se de estudo de caso-controle e não podemos estimar a incidência.

A razão de produtos cruzados é também conhecida como "odds ratio" (OR).

Para o presente trabalho os autores, utilizando ambas as alternativas, para efeito de cálculo, observaram que não houve diferenças significativas usando RR ou OR.

Para o cálculo do risco atribuível percentual foi utilizada a fórmula de Levin ${ }^{2}, \operatorname{RAP}=\frac{f(R R-1)}{f(R R-1)+1} \times 100$, onde $f$ é a freqüència do fator na população e RR é o risco relativo.
Os dados que fazem parte do presente trabalho foram obtidos de trabalho realizado anterior. mente 1 .

A cidade de Porto Alegre foi dividida em quatro setores (central, intermediário norte, intermediário sul e periférico). Em cada um destes setores foram delimitadas as áreas faveladas. A população estudada corresponde a $65,1 \%$ da população total do município e ficou composta de $86,1 \%$ de não favelados e $13,9 \%$ de favelados. Se consideramos somente a população menor de um ano, a proporção de favelados sobe para $18,6 \%$ (Tabela 1 ).

\section{TABELA 1}

Distribuição da população menor de um ano, favelada e não favelada, segundo setores pertencentes ou não à zona de estudo, Porto Alegre, 1980

\begin{tabular}{lcccccc}
\hline \multirow{2}{*}{ Setopulação } & \multicolumn{2}{c}{ Favelada } & \multicolumn{2}{c}{ Não Favelada } & \multicolumn{2}{c}{ Total } \\
\cline { 2 - 7 } & Nọ & $\%$ & No & $\%$ & No & $\%$ \\
\hline Central & 38 & 0,9 & 3.951 & 99,1 & 3.989 & 100,0 \\
Int. Norte & 743 & 13,6 & 4.728 & 86,4 & 5.471 & 100,0 \\
$\begin{array}{l}\text { Int. Sul } \\
\text { Periférico }\end{array}$ & 1.090 & 27,9 & 2.816 & 72,1 & 3.906 & 100,0 \\
\hline $\begin{array}{l}\text { Total Área } \\
\text { de Estudo }\end{array}$ & 1.229 & 36,9 & 2.104 & 63,1 & 3.333 & 100,0 \\
\hline $\begin{array}{l}\text { Fora da Área } \\
\text { de Estudo }\end{array}$ & 3.100 & 18,6 & 13.599 & 81,4 & 16.699 & 100,0 \\
\hline Porto Alegre & 2.129 & 23,4 & 6.955 & 76,6 & 9.084 & 100,0 \\
\hline
\end{tabular}

Fonte: Secretaria de Planejamento. Prefeitura Municipal de Porto Alegre - RS, 1980. 
FISCHMANN, A. \& GUIMARÃES, J.J. đe L. Risco de morrer no primeiro ano de vida entre favelados e não favelados no município de Porto Alegre, RS (Brasil), em 1980. Rev. Saúde públ., S. Paulo, $20: 219-26,1986$.

A distribuição dos óbitos infantis e fetais está apresentada na Tabela 2.

\section{TABELA 2}

Distribuição dos óbitos infantis e fetais ocorridos. entre favelados e não favelados segundo setores da zona de estudo de Porto Alegre, 1980.

\begin{tabular}{|c|c|c|c|c|c|c|c|c|c|c|c|c|c|c|c|}
\hline \multirow{3}{*}{ Setor } & \multicolumn{9}{|c|}{ Obitos Menores de Um Ano } & \multirow{2}{*}{\multicolumn{3}{|c|}{ Total }} & \multirow{2}{*}{\multicolumn{3}{|c|}{ Obitos Fetais }} \\
\hline & \multicolumn{3}{|c|}{$0 \vdash 7 \mathrm{~d}}$. & \multicolumn{3}{|c|}{$7 \vdash 28 \mathrm{~d}$} & \multicolumn{3}{|c|}{$28 \vdash 365 \mathrm{~d}$. } & & & & & & \\
\hline & $\overline{\mathrm{F}}$ & $\tilde{\mathbf{N}} \mathrm{F}$ & $\mathrm{T}$ & $\mathrm{F}$ & $\tilde{\mathrm{N}} \mathrm{F}$ & $T$ & $\mathrm{~F}$ & $\tilde{\mathbf{N} F}$ & $\bar{T}$ & $\mathrm{~F}$ & $\overline{\mathbf{N}} \mathrm{F}$ & $\mathrm{T}$ & $\mathrm{F}$ & $\overline{\mathbf{N} F}$ & $\mathrm{~T}$ \\
\hline Central & 2 & 47 & 49 & - & 11 & 11 & 1 & 25 & 26 & 3 & 83 & 86 & - & 39 & 39 \\
\hline Int. Norte & 13 & 67 & 80 & 5 & 6 & 11 & 37 & 42 & 79 & 55 & 115 & 170 & 26 & 58 & 84 \\
\hline Int. Sul & 26 & 40 & 66 & 13 & 8 & 21 & 49 & 23 & 72 & 88 & 71 & 159 & 42 & 35 & 77 \\
\hline Periférico & 29 & 30 & 59 & 9 & 12 & 21 & 50 & 21 & 71 & 88 & 63 & 151 & 51 & 23 & 74 \\
\hline $\begin{array}{l}\text { Area de } \\
\text { Estudo }\end{array}$ & 70 & 184 & 254 & 27 & 37 & 64 & 137 & 111 & 248 & 234 & 332 & 566 & 119 & 155 & 274 \\
\hline $\begin{array}{l}\text { Fora da } \\
\text { Área de } \\
\text { Estudo }\end{array}$ & $\ldots$ & $\ldots$ & 160 & $\ldots$ & $\ldots$ & 48 & $\ldots$ & $\ldots$ & 166 & $\ldots$ & $\ldots$ & 374 & $\ldots$ & $\ldots$ & 166 \\
\hline $\begin{array}{l}\text { Porto } \\
\text { Alegre }\end{array}$ & $\ldots$ & $\cdots$ & 414 & $\ldots$ & $\ldots$ & 112 & $\ldots$ & $\ldots$ & 414 & $\ldots$ & $\ldots$ & 940 & $\ldots$ & $\ldots$ & 440 \\
\hline
\end{tabular}

Fonte: Secretaria da Saúde e do Meio Ambiente - RS - 1984

A distribuição da mortalidade infantil, neonatal e infantil tardia, de acordo com setores é apresentada na Tabela 3.

\section{TABELA 3}

Coeficiente de mortalidade infantil (neonatal e infantil tardia)

e perinatal, dentro e fora dos limites da zona de estudo, Porto Alegre, 1980.

\begin{tabular}{|c|c|c|c|c|c|c|c|}
\hline \multirow[b]{2}{*}{ Setor } & \multicolumn{4}{|c|}{ Coeficiente de Mortalidade } & \multicolumn{2}{|l|}{ * } & \multirow{2}{*}{$\begin{array}{c}\text { Coeficiente } \\
\text { DI de Mortalidade JE } \\
\text { Perinatal * }\end{array}$} \\
\hline & Neonatal & $\%$ & $\begin{array}{c}\text { Infantil } \\
\text { tardia }\end{array}$ & $\%$ & Infantil & $\%$ & \\
\hline Central & 15,0 & 69,8 & 6,5 & 30,2 & 21,5 & 100,0 & 21,8 \\
\hline Int. Norte & 16,6 & 53,5 & 14,5 & 46,5 & 31,1 & 100,0 & 29,3 \\
\hline Int. Sul & 22,3 & 54,7 & 18,4 & 45,3 & 40,7 & 100,0 & 35,7 \\
\hline Periférico & 24,0 & 53,0 & 21,3 & 47,0 & 45,3 & 100,0 & 38,7 \\
\hline $\begin{array}{l}\text { Area de } \\
\text { Estudo }\end{array}$ & 19,0 & 56,2 & 14,8 & 43,8 & 33,9 & 100,0 & 31,0 \\
\hline $\begin{array}{l}\text { Fora da } \\
\text { Area de } \\
\text { Estudo }\end{array}$ & 22,9 & 55,6 & 18,3 & 44,4 & 41,2 & 100,0 & 35,1 \\
\hline $\begin{array}{l}\text { Porto } \\
\text { Alegre }\end{array}$ & 20,4 & 56,0 & 16,0 & 44,0 & 36,4 & 100,0 & 32,5 \\
\hline
\end{tabular}

Fonte: Secretaria da Saúde e do Meio Ambiente - RS - 1984

* Coeficiente por mil menores de um ano 
FISCHMANN, A. \& GUIMARÃES, J.J. de L. Risco de morrer no primeiro ano de vida entre favelados e não favelados no município de Porto Alegre, RS (Brasil), em 1980. Rev. Saúde públ., S. Paulo, $20: 219$-26, 1986.

A distribuição do coeficiente de mortalidade infantil (CMI) por favelados e não favelados, em cada um dos setores, é apresentada na Tabeia 4.

\section{TABELA 4}

Coeficiente de mortalidade infantil entre favelados e não favelados, segundo setores da zona de estudo, Porto Alegre, 1980

\begin{tabular}{lccc}
\hline \multirow{2}{*}{ Setor } & \multicolumn{2}{c}{$\begin{array}{c}\text { Coeficiente de Mortalidade de Infantil* } \\
1.000 \text { Habitantes / Um Ano }\end{array}$} & \multirow{2}{*}{ Total } \\
\cline { 2 - 3 } & Favelados & Não Favelados & \\
\hline Central & 78,9 & 21,0 & 21,5 \\
Int. Norte & 74,0 & 24,3 & 31,1 \\
Int. Sul & 80,7 & 25,2 & 40,7 \\
Periférico & 71,6 & 30,0 & 45,3 \\
Coeficiente da área de estudo & 75,5 & 24,4 & 33,9 \\
\hline
\end{tabular}

Fonte: Secretaria da Saúde e do Meio Ambiente - RS - 1984

A distribuição dos coeficientes de mortalidade e a classificação pelos cinco mais importantes grupos de causas para toda a área de estudo e para cada setor é apresentada (respectivamente) na Tabela 5.

\section{TABELA 5}

Classificação dos cinco mais importantes grupos

de causas de mortalidade infantil entre favelados e não favelados de Porto Alegre, 1980

\begin{tabular}{lrrrrrrrrr}
\hline \multirow{2}{*}{$\begin{array}{c}\text { População Grupo } \\
\text { de Causa }\end{array}$} & \multicolumn{3}{c}{ Favelado } & \multicolumn{4}{c}{ Não Favelado } & \multicolumn{3}{c}{ Total } \\
\cline { 2 - 10 } & \multicolumn{1}{c}{ Coef. ${ }^{*}$} & Classif. & \multicolumn{1}{c}{$\%$} & Coef. ${ }^{*}$ & Classif. & $\%$ & Coef. * Classif. \\
\hline Afecções perinatais & 28,2 & 21,3 & 29 & 47,6 & 11,6 & 19 & 39,6 & 13,4 & 19 \\
Pneumonia e Gripe & 28,6 & 21,6 & 19 & 14,8 & 3,6 & 39 & 20,5 & 6,9 & 29 \\
Anomalias Congênitas & 4,7 & 3,5 & 59 & 16,9 & 4,1 & 29 & 11,8 & 4,0 & 39 \\
Doenças Inf. Intestinais & 13,7 & 10,3 & 39 & 7,2 & 1,8 & 49 & 9,9 & 3,3 & 49 \\
Septicemia & 8,5 & 6,5 & 49 & 3,3 & 0,8 & 59 & 5,5 & 1,8 & 59 \\
\hline Outros Grupos & 16,3 & 12,3 & - & 10,2 & 2,5 & - & 12,7 & 4,5 & - \\
\hline Total & 100,0 & 75,5 & - & 100,0 & 24,4 & - & 100,0 & 33,9 & - \\
\hline
\end{tabular}

Fonte: Secretaria da Saúde e do Meio Ambiente - RS, 1984.

* Coeficiente por mil menores de um ano 
A distribuição dos óbitos por causas e por setores é apresentada na Tabela 6.

TABELA 6

Cinco principais grupos de causas de óbitos, no primeiro ano de vida, em favelados e não favelados, de acordo com os setores onde ocorreram, em $65 \%$ da população de Porto Alegre - RS, 1980

\begin{tabular}{|c|c|c|c|c|c|c|c|c|c|c|c|c|c|c|c|}
\hline \multirow{2}{*}{$\begin{array}{ll} & \text { Setor } \\
\text { Causa }\end{array}$} & \multicolumn{3}{|c|}{ Central } & \multicolumn{3}{|c|}{$\begin{array}{c}\text { Intermediário } \\
\text { Norte }\end{array}$} & \multicolumn{3}{|c|}{$\begin{array}{c}\text { Intermediário } \\
\text { Sul }\end{array}$} & \multicolumn{3}{|c|}{ Periférico } & \multicolumn{3}{|c|}{ Todos } \\
\hline & $\overline{\mathbf{N} F a v}$ & Fav & $\mathrm{T}$ & $\widehat{\mathrm{NF}} \mathrm{Fav}$ & Fav & $\mathrm{T}$ & NFav & Fav & $T$ & NFav & Fav & $T$ & $\widehat{\text { NFav }}$ & Fav & $T$ \\
\hline $\begin{array}{l}\text { Pneumonia e } \\
\text { Gripe }\end{array}$ & 11 & 1 & 12 & 15 & 21 & 36 & 9 & 24 & 33 & 14 & 21 & 35 & 49 & 67 & 116 \\
\hline $\begin{array}{l}\text { Afecções } \\
\text { originadas } \\
\text { no período } \\
\text { perinatal }\end{array}$ & 36 & 2 & 38 & 53 & 13 & 66 & 45 & 26 & 71 & 24 & 25 & 49 & 158 & 66 & 224 \\
\hline $\begin{array}{l}\text { Doença } \\
\text { infecciosa } \\
\text { intestinal }\end{array}$ & 6 & - & 6 & 8 & 4 & 12 & 5 & 14 & 19 & 5 & 14 & 19 & 24 & 32 & 56 \\
\hline Septicemia & 5 & - & 5 & 3 & 4 & 7 & 1 & 7 & 8 & 2 & 9 & 11 & 11 & 20 & 31 \\
\hline $\begin{array}{l}\text { Anomalias } \\
\text { congênitas }\end{array}$ & 19 & - & 19 & 22 & 3 & 25 & 7 & 6 & 13 & 8 & 2 & 10 & 56 & 11 & 67 \\
\hline Outras & 6 & - & 6 & 14 & 10 & 24 & 4 & 11 & 15 & 10 & 17 & 27 & 34 & 38 & 72 \\
\hline Total & 83 & 3 & 86 & 115 & 55 & 170 & 71 & 88 & 159 & 63 & 88 & 151 & 332 & 234 & 566 \\
\hline
\end{tabular}

Fonte: Secretaria da Saúde e do Meio Ambiente - RS - 1984.

Segundo Walter ${ }^{5}$, com a finalidade de obter um intervalo de confiança de $95 \%$ e assumindo que a distribuição é normal, pode-se calcular a variância do risco atribuivel percentual (RAP) através da fórmula:

$$
\mathrm{V}(\mathrm{RAP})=\left[\frac{\mathrm{cn_{2 }}}{\mathrm{dn_{1 }}}\right]^{2} \mathrm{X}\left[\frac{\mathrm{a}}{\mathrm{cn_{1 }}}+\frac{\mathrm{b}}{\mathrm{dn_{2 }}}\right]
$$

Para o cálculo de risco relativo, com a finalidade de obter um intervalo de confiança de $95 \%$, calculou-se o qui-quadrado e utilizou-se a fórmula $\operatorname{RR} \quad[1 \pm(1,96 / X)]$, para obter a variância.

\section{RESULTADOS}

1. Cálculo de RR e RAP, para a área total de estudo (dados oriundos da Tabela 2).

\begin{tabular}{crrr} 
Fator de Risco & \multicolumn{2}{c}{ Obito Infantil } & Total \\
Favelado & Sim & \multicolumn{1}{c}{ Não } & \\
Sim & 234 & 2.866 & 3.100 \\
Não & 332 & 13.267 & 13.599 \\
Total & 566 & 16.133 & 16.699
\end{tabular}

Cálculo do RR

$$
\begin{aligned}
& \mathrm{RR}=3,1 \\
& \mathrm{OR}=3,26 \\
& \chi^{2}=201,08 \\
& \chi=\sqrt{201,08}=14,2 \\
& \operatorname{RR}[1 \pm(1,96 / \chi)] \\
& \operatorname{RR}[1 \pm(1,96 / 14,2)] \\
& \operatorname{RR} \in(2,47 ; 3,62) \rightarrow 95 \%
\end{aligned}
$$

\section{Cálculo do RAP}

$$
\begin{aligned}
& \text { RAP }=28,1 \% \text { para } f=0,186 \text { (Tab. 1) } \\
& \mathrm{V}(\mathrm{RAP})=0,0006404 \\
& \mathrm{DP}=\sqrt{0,0006404} \\
& \mathrm{DP}=0,0253061 \\
& (\mathrm{RAP})=28,1 \pm(1,96) \times(2,5) \\
& (\mathrm{RAP}) \in[23,2 ; 33] \rightarrow 95 \%
\end{aligned}
$$

\section{Interpretação para RAP}

- Vinte e oito por cento $(23,2$ - $33 \%)$ da mortali. dade infantil da área em estudo está associada a 
FISCHMANN, A. \& GUIMARÃES, J.J. de L. Risco de morrer no primeiro ano de vida entre favelados e não favelados no município de Porto Alegre, RS (Brasil), em 1980. Rev. Saúde públ., S. Paulo, $20: 219-26,1986$.

freqüência do fator favelado em $18,6 \%$ da popu. lação menor de um ano, com um intervalo de $95 \%$ de confiança, ou

- Vinte e oito por cento $(23,2$ - 33\%) da mortalidade infantil da área é atribuível ao fato do menor de um ano ser favelado, com um intervalo de $95 \%$ de confiança.

Calculou-se também o risco relativo para o coeficiente de mortalidade neonatal $(1,9)$ e para o coeficiente de mortalidade infantil tardia $(5,4)$, revelando que nos favelados o risco de morrer dos 28 dias aos 11 meses é 5,4 vezes maior que nos não favelados.

2. Cálculo de RR e RAP de acordo com os setores, para mortalidade em menores de um ano (dados obtidos das Tabelas 3 e 4 )

$\begin{array}{lcccrc}\text { Setor } & \text { RR } & 95 \% & \text { f }^{*} & \begin{array}{r}\text { RAP } \\ (\%)\end{array} & \begin{array}{l}\text { V (RAP) } \\ (\%)\end{array} \\ \text { Central } & 4,0 & (1,3 ; 5,8) & 0,009 & 2,4 & (-1,6 ; 6,3) \\ \text { Int. Norte } & 3,2 & (2,2 ; 4,4) & 0,139 & 21,7 & (13,5 ; 29,9) \\ \text { Int. Sul } & 3,2 & (2,3 ; 4,5) & 0,28 & 38,1 & (27,3 ; 48,9) \\ \text { Periférico } & 2,4 & (1,2 ; 4,9) & 0,37 & 33,8 & (21,5 ; 40,1)\end{array}$

$\mathrm{f} *$ (percentual de favelados obtido da Tabela 1)

O setor que apresentou maior percentual de favelados foi o periférico $(37,0 \%)$, no entanto o RAP mais alto foi observado no setor intermediário Sul $(38,1 \%)$ com $28,0 \%$ de favelados. Este fato explicase pelo alto RR observado neste setor. O RR mais baixo no setor periférico justifica-se pelo tato de não existir diferenças tão acentuadas entre favelados e não favelados como nos demais setores. O baixo RAP observado no setor central $(2,4 \%)$ decorre do pequeno percentual de favelados ali residentes $(0,9 \%)$.

\section{Cálculo do RR e RAP de acordo com os cinco} mais importantes grupos de causas. Ver Tabela 7 (a partir dos dados da Tabela 6).

Para o grupo Anomalias Congênitas, como causa de óbito, observou-se que não ocorreram óbitos no setor central. No intermediário norte, no periférico e na média para todos os setores o RR foi menor do que um. Neste caso, invertendo-se a tabela e considerando o fato de não ser favelado como risco de óbito por anomalia congênita, obteremos o seguinte para todos os setores:

\begin{tabular}{lccr}
\multicolumn{1}{c}{$\begin{array}{l}\text { Anomalia } \\
\text { Congênita }\end{array}$} & Sim & Não & Total \\
Não Favelado & 56 & 13.543 & 13.599 \\
Favelado & 11 & 3.089 & 3.100 \\
Total & 67 & 16.632 & 16.699 \\
Então, RR & $=1,2 \in(0,5 ; 2,65) \rightarrow-95 \%$ & \\
$\qquad$ RAP & $=13,9 \%$ para $\mathrm{f}=0,81$ &
\end{tabular}

Ou seja, o risco relativo de não sendo favelado morrer de anomalia congênita de 1,2 vezes foi considerado não significativo, estatisticamente.

TABELA 7

Risco relativo e risco atribuível ao fator favelado no primeiro ano de vida, de acordo com os cinco primeiros grupos de causas e setor onde ocorreram, Porto Alegre, RS, 1980

\begin{tabular}{|c|c|c|c|c|c|c|c|c|c|c|}
\hline \multirow[b]{2}{*}{ Favelados } & \multicolumn{2}{|c|}{ Central } & \multicolumn{2}{|c|}{ Int. Norte } & \multicolumn{2}{|c|}{ Int. Sul } & \multicolumn{2}{|c|}{ Periférico } & \multicolumn{2}{|c|}{ Todos os Setores } \\
\hline & $\mathbf{R} \mathbf{R}$ & $\mathrm{RA}(\%)$ & $\mathbf{R R}$ & $\mathrm{RA}(\%)$ & $\mathbf{R R}$ & $\operatorname{RA}(\%)$ & $\mathbf{R R}$ & RA (\%) & $\mathbf{R R}$ & RA (\%) \\
\hline $\begin{array}{l}\text { Afeçõos Originadas } \\
\text { do Período Perinatal }\end{array}$ & $\begin{array}{c}5,5 \\
(1,6 ; 18,9)\end{array}$ & $\begin{array}{c}7,3 \\
(0,24 ; 14,4)\end{array}$ & $\begin{array}{c}1,6 \\
(0,9 ; 2,8)\end{array}$ & $\begin{array}{c}7,5 \\
(4 ; 10)\end{array}$ & $\begin{array}{c}1,5 \\
(0,92 ; 2,42)\end{array}$ & $\begin{array}{l}12,2 \\
\text { NS }\end{array}$ & $\begin{array}{c}1,8 \\
(1,01 ; 2,2)\end{array}$ & $\begin{array}{c}22,8 \\
(11,7 ; 33,9)\end{array}$ & $\begin{array}{c}1,8 \\
(1,4 ; 2,4)\end{array}$ & $\begin{array}{c}12,8 \\
(5,6 ; 20,0)\end{array}$ \\
\hline Pneumonia e Gripe & $\begin{array}{c}9,7 \\
(1,7 ; 53,8)\end{array}$ & $\begin{array}{c}8,0 \\
(4,4 ; 11,6)\end{array}$ & $\begin{array}{c}4,6 \\
(2,4 ; 8,8)\end{array}$ & $\begin{array}{c}32,8 \\
(14,2 ; 51,4)\end{array}$ & $\begin{array}{c}7,0 \\
(3,6 ; 13,6)\end{array}$ & $\begin{array}{c}63,6 \\
(52,9 ; 74,3)\end{array}$ & $\begin{array}{c}2,6 \\
(1,3 ; 5,1)\end{array}$ & $\begin{array}{c}37,1 \\
(24 ; 50,2)\end{array}$ & $\begin{array}{c}6,1 \\
(4,6 ; 8,2)\end{array}$ & $\begin{array}{c}48,7 \\
37,6 ; 59,8)\end{array}$ \\
\hline $\begin{array}{l}\text { Doença Infecciosa } \\
\text { Intestinal }\end{array}$ & (1) - & (1) - & $\begin{array}{c}3,3 \\
(1,02 ; 10,63)\end{array}$ & $\begin{array}{c}23,8 \\
(9,8 ; 37,8)\end{array}$ & $\begin{array}{c}7,3 \\
(3,0 ; 17,6)\end{array}$ & $\begin{array}{c}63,7 \\
(36,7 ; 99,9)\end{array}$ & $\begin{array}{c}4,8 \\
(1,9 ; 12,2)\end{array}$ & $\begin{array}{c}58,4 \\
(25,8 ; 91)\end{array}$ & $\begin{array}{c}5,9 \\
(3,9 ; 9,0)\end{array}$ & $\begin{array}{c}47,7 \\
(32,1 ; 63,3)\end{array}$ \\
\hline Septicemia & (1) - & (1) - & $\begin{array}{c}8,5 \\
(2,4 ; 29,2)\end{array}$ & $\begin{array}{c}50,5 \\
(8,2 ; 92,8)\end{array}$ & $\begin{array}{c}18,2 \\
(4,0 ; 81,3)\end{array}$ & $\begin{array}{c}82,7 \\
(50,9,100,00)\end{array}$ & $\begin{array}{c}7,7 \\
(2,1 ; 28,0)\end{array}$ & $\begin{array}{c}71,2 \\
(34,6 ; 100,0)\end{array}$ & $\begin{array}{c}8,0 \\
(4,3 ; 14,8)\end{array}$ & $\begin{array}{c}56,7 \\
(29,82 ; 83,58)\end{array}$ \\
\hline $\begin{array}{l}\text { Anomalias } \\
\text { Congênitas }\end{array}$ & (1) - & (1) - & 0,9 & (2) & $\begin{array}{c}2,2 \\
\text { (NS ) }\end{array}$ & $\begin{array}{c}35,8 \\
(-16 ; 73,2)\end{array}$ & 0,4 & (2) & 0,9 & (2) \\
\hline
\end{tabular}


FISCHMANN, A. \& GUIMARĀES, J.J. de L. Risco de morrer no primeiro ano de vida entre favelados e não favelados no município de Porto Alegre, RS (Brasil), em 1980. Rev. Saúde públ., S. Paulo, $20: 219-26,1986$.

4. Risco de mortalidade fetal la partir dos dados das Tabelas 1 e 2).

$\begin{array}{lcrc}\begin{array}{l}\text { Mortalidade } \\ \text { Fetal }\end{array} & \text { Sim } & \text { Não } & \text { Total } \\ & & & \\ \text { Favelado } & 119 & 3.100 & 3.219 \\ \text { Não Favelado } & 115 & 13.599 & 13.754 \\ \text { Total } & 274 & 16.699 & 16.973(1)\end{array}$

(1) estimativa de partos a termo da área de estudo

$R R=4,5 \in[3,5 ; 5,8] \rightarrow 95 \%$

RAP $=24,7 \%$, considerando $f=0,139$

(RAP) $\in[18,4 ; 30,9] \rightarrow 95 \%$

\section{COMENTÁRIOS E CONCLUSÕES}

$O$ presente trabalho quantificou as desigualdades da mortalidade infantil entre favelados e não favelados ocorrida em $65 \%$ da população de Porto Alegre em 1980, através de cálculo do risco relativo e do risco atribuivel percentual.

Em todos os setores estudados, o RR de ser favelado e morrer no primeiro ano de vida foi em média 3,1 vezes maior que o fato de não ser favelado; dependendo do setor, o risco foi de 2,4 (periférico) e de 4,0 (setor central) e nos outros dois setores foi 3,2. O RAP médio, de $28 \%$, variou de 21,7 (setor intermediário norte) a 38,1 (setor intermediário sul), considerados significativos estatisticamente. No setor central, o RAP de 2,4 não foi significativo estatisticamente; o baixo percentual de favelados neste setor $(0,9 \%)$, seguramente influiu para isso.

O risco relativo por grupo de cinco principais causas, com exceção dos óbitos por anomalias congênitas, foi sempre maior para o grupo favelado e variou entre 1,8 (afeç̧ōes perinatais), 5,9 (doença infecciosa intestinal), 6,1 (pneumonia e gripe) e 8,0 (septicemia) (Tabela 7).
Quanto ao risco de morrer por anomalias congênitas esperava-se que fosse maior para o grupo favelado, no entanto ele foi de 1,2 para o não favela. do e considerado não significante ao nivel de $95 \%$ de confiança.

Presume-se, pois, que esta patologia não aparece predominantemente no favelado, ou:

a) por ser o risco relativo de mortalidade fetal 4,5 vezes maior nos favelados, um grande número de crianças faveladas que poderiam ter anomalia congênita, nem sequer teriam nascido vivas;

b) o diagnóstico de anomalia congênita foi omitido como causa básica face ao alto risco de morrer por outras causas (pneumonia, doença infecciosa intestinal e septicemia).

Estes fatos sugerem investigações posteriores.

Quanto ao RAP pode-se dizer que variou amplamente de acordo com os setores e com as causas. 0 menor RAP encontrado foi de $12,8 \%$ (mortalidade perinatal) e o maior foi de $56,7 \%$ para septicemia em todos os setores; no intermediário sul o RAP por septicemia foi de $82,7 \%$, visto que houve somente um óbito por esta causa em não favelados (Tabela 7).

Outras causas também foram atribuiveis ao fator favelado, como o RAP de $47,7 \%$ para doença infecciosa intestinal e de $48,7 \%$ para pneumonia e gripe, e que aumentam quando se individualiza por setor (Tabela 7).

Algumas das cifras são alarmantes e localizam o problema em termos de prioridade. A par do problema social que acompanha as piores taxas, chama a atenção a necessidade de se verificar qual a acessibilidade e a qualidade de assistência à saúde posta à disposição da população favelada, pois muitas destas causas podem ser reduzidas com cuidados médicos adequados.

FISCHMANN, A. \& GUIMARĀES, J.J. de L. [Infant mortality risk in shantytown and non-shantytown residents in the city of Porto Alegre, Rio Grande do Sul State, Brazil, 1980] Rev.Saúde públ., S.Paulo, $20: 219-26,1986$.

ABSTRACT: The calculation of infant mortality relative risk (RR) and attributable risk percent (ARP), related to shantytown residents (SR) in Porto Alegre, Rio Grande do Sul State, Brazil, 1980 is non-shantytown, residents (NSR). The infant mortality attributable risk due to SR with $95 \%$ probability, ranged from 23.2 to 33.0 , if other risk factors were not considered. RR ranged from 2.4 to 3.6 . For the main five causes of death RR was always higher for SR, except for congenital anomalies, where the relative risk was higher for NSR. RR was: 1.8 times higher for certain conditions originated in pregnancy and around birth (COPAB), 5.9 for intestinal infections diseases (IID), 6.1 for pneumonia and influenza (PI) and 8.0 for septicemia. As for ARP, it was found that $28 \%$ of infant mortality occurred in SR, which corresponded to $18.6 \%$ of the total population. Considering the main causes of death in SR, ARP was $12.8 \%, 47.7 \%, 48.7 \%$ and $56.7 \%$ respectively for COPAB, IID, PI and speticemia. Even if we take into account that conditions for people living in slums are determined by social and economical factors, it was recommended an evaluation of health care services seeing that such causes of deaths could be prevented by adequate management health care programs, directed mainly to people living under the worst conditions.

UNITERMS: Risk. Infanty mortality. Morbidity. Socioeconomic factors. 
FISCHMANN, A. \& GUIMARÃES, J.J. de L. Risco de morrer no primeiro ano de vida entre favelados e não favelados no município de Porto Alegre, RS (Brasii), em 1980. Rev. Saúde públ., S. Paulo, $20: 219-26,1986$.

\section{REFERENCIAS BIBLIOGRAFICAS}

1. GUIMARĀES, J.J.L. \& FISCHMANN, A. Inequalities in 1980 infant mortality among Shantytown residents and Non-Shanty town residents in the municipality of Porto Alegre, Rio Grande do Sul, Brazil. Bull.Pan Amer.Hith Org., 19 (3), 1985 [ no prelo]

2. LEVIN, M.L. apud LEVITON, A. Definitions of attributable risk: letter to the editor. Amer.J.Epidem, 98: $231,1973$.

3. LILIENFELD, A.M. \& LILIENFELD, D.E. Foundations of epidemiology. Oxford, Oxford University Press, 1980. p. 209.
4. SARUE P., H.E. et al. O conceito de risco e a programação dos cuidados à saúde: manual básico de aprendizagem inicial. Brasília?, 1984. [Tradução adaptada: Instituto Interamericano del Niño, Publ. Cient., 883 e Centro Latinoamericano de Perinatologia y Desarrollo Humano, Publ. Cient. 1007].

5. WALTER, S.D. Calculation of attributable risks from epidemiological data. Int.J.Epidem., 7: 175-82, 1978.

Recebido para publicação em 02/09/1985

Reapresentado em 03/01/1986

Aprovado para publicação em 03/02/1986 